\title{
A Mathematical Model of the Evaporation of a Thin Sessile Liquid Droplet: Comparison Between Experiment and Theory
}

\author{
G. J. Dunn ${ }^{\text {a }}$, S. K. Wilson ${ }^{\mathrm{a}, *}$, B. R. Duffy ${ }^{\mathrm{a}}$, S. David ${ }^{\mathrm{b}}$, \\ K. Sefiane ${ }^{b}$ \\ ${ }^{a}$ Department of Mathematics, University of Strathclyde, Livingstone Tower, \\ 26 Richmond Street, Glasgow G1 1XH, UK \\ ${ }^{\mathrm{b}}$ School of Engineering and Electronics, University of Edinburgh, Sanderson \\ Building, The King's Buildings, Mayfield Road, Edinburgh EH9 3JL, UK
}

\begin{abstract}
A mathematical model for the quasi-steady diffusion-limited evaporation of a thin axisymmetric sessile droplet of liquid with a pinned contact line is formulated and solved. The model generalises the theoretical model proposed by Deegan et al. [Phys. Rev. E, 62 (2000) 756-765] to include the effect of evaporative cooling on the saturation concentration of vapour at the free surface of the droplet, and the dependence of the coefficient of diffusion of vapour in the atmosphere on the atmospheric pressure. The predictions of the model are in good qualitative, and in some cases also quantitative, agreement with recent experimental results. In particular, they capture the experimentally observed dependence of the total evaporation rate on the thermal conductivities of the liquid and the substrate, and on the atmospheric pressure.
\end{abstract}

Key words: Evaporation; Liquid Droplet; Evaporative Cooling; Atmospheric Pressure.

\section{Introduction}

The evaporation of liquid droplets is of fundamental importance in a huge variety of practical situations ranging from technological applications such as

\footnotetext{
* Corresponding author. Tel.: + 44 (0) 141548 3820; fax: + 44 (0) 1415483345.

Email addresses: s.k.wilson@strath.ac.uk (S. K. Wilson),

b.r.duffy@strath.ac.uk (B. R. Duffy), k.sefiane@ed.ac.uk (K. Sefiane).
} 
ink-jet printing, spray cooling and various coating processes, to a variety of biological and geophysical contexts. As a result droplet evaporation has been the subject of considerable theoretical and experimental research in recent years. Significant recent papers include those by Deegan [1], Deegan et al. [2], Hu and Larson [3-5], Popov [6], Poulard, Guéna and Cazabat [7], Sultan, Boudaoud and Ben Amar [8], Shahidzadeh-Bonn et al. [9], and Girard et al. [10].

Physical experiments conducted recently by David, Sefiane and Tadrist [11] using a variety of liquids and substrates show that the thermal conductivities of the liquid and the substrate, and the atmospheric pressure can have a significant effect on the total evaporation rate. Neither of these effects is captured by the widely used pioneering theoretical model proposed by Deegan et al. [2] (hereafter referred to simply as "the Deegan model" for brevity).

In this paper a mathematical model for the quasi-steady diffusion-limited evaporation of a thin axisymmetric sessile droplet of liquid with a pinned contact line is formulated and solved. This model generalises the Deegan model to include the effect of evaporative cooling on the saturation concentration of vapour at the free surface of the droplet, and the dependence of the coefficient of diffusion of vapour in the atmosphere on the atmospheric pressure. For simplicity, the present initial model is, however, restricted to the special case of thin droplets with small contact angles. The results presented here show that the predictions of the model are in good qualitative, and in some cases also quantitative, agreement with the experimental results. In particular, they capture the experimentally observed dependence of the total evaporation rate on the thermal conductivities of the liquid and the substrate, and on the atmospheric pressure.

The present paper describes some aspects of the work presented at the International Workshop on Bubble and Drop Interfaces held on 25th-28th March 2007 in Granada, Spain. A preliminary account of part of this work was given by Dunn et al. [12].

\section{The Mathematical Model}

Figure 1 near here.

Consider the quasi-steady diffusion-limited evaporation of a thin axisymmetric sessile droplet of liquid with constant density $\rho$, surface tension $\sigma$, and thermal conductivity $k$ on a thin horizontal substrate of constant thickness $h^{\mathrm{s}}$ with constant thermal conductivity $k^{\mathrm{s}}$. Referred to cylindrical polar coordinates $(r, \phi, z)$ with origin on the substrate at the centre of the droplet and with 
the $z$ axis vertically upwards, the shape of the free surface of the droplet is denoted by $z=h(r, t)$, the upper surface of the substrate by $z=0$, and the lower surface of the substrate by $z=-h^{\mathrm{s}}$, as shown in Fig. 1.

The contact lines of evaporating droplets are typically pinned by surface roughness (or other) effects during the first stage of their evaporation, but can de-pin prior to complete evaporation. All of the experimental results for temperature and evaporation rate reported by David et al. [11] are for droplets in this first (pinned) stage, and so in the present model we assume that the droplet radius $R$ remains constant. We also assume that the droplet is sufficiently small that surface tension effects dominate gravitational effects, and that the droplet is sufficiently thin (in particular, that the contact angle $\theta=\theta(t)$ is sufficiently small) that it has the simple quasi-static parabolic shape

$$
h=\frac{\theta\left(R^{2}-r^{2}\right)}{2 R}
$$

with volume $V=V(t)$ given by

$$
V=\frac{\pi R^{3} \theta}{4}
$$

While the former assumption is well justified for the experiments of David et al. [11], the latter assumption is more questionable (see the discussion about this assumption in Section 4). The total evaporation rate is given by

$$
-\frac{\mathrm{d} V}{\mathrm{~d} t}=\frac{2 \pi}{\rho} \int_{0}^{R} J(r, t) r \mathrm{~d} r
$$

where $J=J(r, t)(>0)$ is the local evaporative mass flux from the droplet.

The atmosphere surrounding the droplet and the substrate is assumed to be at constant atmospheric temperature $T_{\mathrm{a}}$ and pressure $p_{\mathrm{a}}$. Since both the droplet and the substrate are thin, their temperatures, denoted by $T=T(r, z, t)$ and $T^{\mathrm{s}}=T^{\mathrm{s}}(r, z, t)$, satisfy

$$
\frac{\partial^{2} T}{\partial z^{2}}=0, \quad \frac{\partial^{2} T^{\mathrm{s}}}{\partial z^{2}}=0
$$

The mass flux from the droplet satisfies the local energy balance

$$
L J=-k \frac{\partial T}{\partial z}
$$


on $z=h$ for $r<R$, where $L$ is the latent heat of vaporisation. Hence, assuming that both the temperature and the heat flux are continuous between the droplet and the wetted part of the substrate, and that the lower surface of the substrate is at the atmospheric temperature $T_{\mathrm{a}}$, we have

$$
T=T_{\mathrm{a}}-L J\left(\frac{z}{k}+\frac{h^{\mathrm{s}}}{k^{\mathrm{s}}}\right), \quad T^{\mathrm{s}}=T_{\mathrm{a}}-\frac{L J}{k^{\mathrm{s}}}\left(z+h^{\mathrm{s}}\right),
$$

showing clearly the evaporative cooling of both the droplet and the substrate.

Assuming that transport of vapour in the atmosphere is dominated by diffusion (see, for example, Popov [6]), the concentration of vapour in the atmosphere above the droplet and the substrate, denoted by $c=c(r, z, t)$, satisfies Laplace's equation,

$$
\nabla^{2} c=0
$$

Since the droplet is thin, Eq. (7) holds in the half-space $z>0$, and the boundary conditions for $c$ on the free surface of the droplet may be imposed on $z=0$ rather than on $z=h$.

At the free surface of the droplet we assume that the atmosphere is saturated with vapour so that $c=c_{\text {sat }}(T)$, where the saturation concentration $c_{\text {sat }}=$ $c_{\text {sat }}(T)$ is assumed to be a linearly increasing function of temperature given by

$$
c_{\mathrm{sat}}(T)=c_{\mathrm{sat}}\left(T_{\mathrm{a}}\right)+\left.\frac{\mathrm{d} c_{\mathrm{sat}}}{\mathrm{d} T}\right|_{T=T_{\mathrm{a}}}\left(T-T_{\mathrm{a}}\right)
$$

on $z=0$ (rather than on $z=h$ ) for $r<R$. On the dry part of the substrate there is no mass flux, i.e.

$$
\frac{\partial c}{\partial z}=0
$$

on $z=0$ for $r>R$, and far from the droplet the concentration of vapour approaches its ambient value, i.e.

$$
c \rightarrow H c_{\text {sat }}\left(T_{\mathrm{a}}\right)
$$

as $\left(r^{2}+z^{2}\right)^{1 / 2} \rightarrow \infty$, where $H$ is the relative saturation of the atmosphere far from the droplet (which may be zero or non-zero). Once $c$ is known the mass 
flux from the droplet is given by

$$
J=-D \frac{\partial c}{\partial z}
$$

on $z=0$ (rather than on $z=h$ ) for $r<R$, where $D$ is the coefficient of diffusion of vapour in the atmosphere. A standard result from the theory of gases (see, for example, Reid, Prausnitz and Poling [13]) is that $D$ is inversely proportional to pressure, i.e.

$$
D=\frac{D_{\text {ref }} p_{\text {ref }}}{p_{\text {a }}}
$$

where $D_{\text {ref }}$ is the reference value of the coefficient of diffusion at the reference atmospheric pressure $p_{\text {ref }}=998$ mbar. Note that the atmospheric pressure $p_{\text {a }}$ enters the model only via this expression for $D$.

In the special case when the variation of the saturation concentration with temperature is negligible (i.e. when $c_{\text {sat }}(T) \simeq c_{\text {sat }}\left(T_{\mathrm{a}}\right)$ ) a straightforward generalisation of the standard Deegan model with $D$ given by Eq. (12) is recovered. In this case the problem for $c$ given by Eqs $(7)-(10)$ is independent of the temperature, and the solution for $c$ (not repeated here for brevity) is well known and yields

$$
J=\frac{2 D(1-H) c_{\mathrm{sat}}\left(T_{\mathrm{a}}\right)}{\pi \sqrt{R^{2}-r^{2}}}
$$

for $r<R$, and hence

$$
-\frac{\mathrm{d} V}{\mathrm{~d} t}=\frac{4 R D(1-H) c_{\mathrm{sat}}\left(T_{\mathrm{a}}\right)}{\rho}
$$

and so, in particular, the evaporation rate is proportional to the radius of the droplet and inversely proportional to the atmospheric pressure (via $D$ ), but is independent of the thermal conductivities of the liquid and the substrate.

In general, the problem for $c$ given by Eqs (7)-(10) depends on the temperature and has to be solved numerically. This was done using a finite-element method implemented using the MATLAB-based numerical analysis package COMSOL Multiphysics (formerly FEMLAB). The far-field condition was imposed on a notional computational boundary at a distance of $320 R$ from the origin, and the numerical mesh used incorporated a semi-circular subdomain of size $0.1 R$ centred at the contact line $r=R$ with a very much finer mesh in order to resolve the singularity there. 


\begin{tabular}{|c|c|c|c|c|}
\hline Parameter & Units & Acetone & Methanol & Water \\
\hline$\rho$ & $\mathrm{kg} \mathrm{m}^{-3}$ & 788 & 790 & 998 \\
$\sigma$ & $\mathrm{N} \mathrm{m}^{-1}$ & $2.38 \times 10^{-2}$ & $2.23 \times 10^{-2}$ & $7.25 \times 10^{-2}$ \\
$k$ & $\mathrm{~W} \mathrm{~m}^{-1} \mathrm{~K}^{-1}$ & 0.161 & 0.203 & 0.604 \\
$L$ & $\mathrm{~J} \mathrm{~kg}^{-1}$ & $5.49 \times 10^{5}$ & $1.20 \times 10^{6}$ & $2.45 \times 10^{6}$ \\
$c_{\text {sat }}$ & $\mathrm{kg} \mathrm{m}^{-3}$ & 0.637 & 0.186 & $1.94 \times 10^{-2}$ \\
$\mathrm{~d} c_{\text {sat }} / \mathrm{d} T$ & $\mathrm{~kg} \mathrm{~m}^{-3} \mathrm{~K}^{-1}$ & $2.84 \times 10^{-2}$ & $9.47 \times 10^{-3}$ & $1.11 \times 10^{-3}$ \\
$D_{\text {ref }}$ (for air $)$ & $\mathrm{m}^{2} \mathrm{~s}^{-1}$ & $1.06 \times 10^{-5}$ & $1.50 \times 10^{-5}$ & $2.46 \times 10^{-5}$ \\
$D_{\text {ref }}$ (for helium) & $\mathrm{m}^{2} \mathrm{~s}^{-1}$ & $4.26 \times 10^{-5}$ & $5.96 \times 10^{-5}$ & $8.26 \times 10^{-5}$ \\
\hline
\end{tabular}

Table 1

Values of the relevant physical parameters for the three liquids used at temperature $T_{\mathrm{a}}=295 \mathrm{~K}$.

\section{Comparison with Experiments}

David et al. [11] conducted physical experiments using small droplets of three different liquids (specifically, acetone, methanol and water) on thin substrates of thickness $1 \mathrm{~mm}$ made of four different materials (specifically, aluminium, titanium, macor and PTFE). Droplets were deposited on the substrates and left to evaporate spontaneously. The substrates were coated with a very thin $(3 \mu \mathrm{m})$ layer of $\mathrm{Al}$ to ensure that they had the same surface energy and roughness properties without significantly altering their thermal properties. In particular, the initial static contact angles of the droplets when they were first deposited onto the substrates were approximately $\theta(0)=43^{\circ}$ for acetone and methanol, and $\theta(0)=60^{\circ}$ for water. The evaporation rates were measured using a KRUSS DSA 100 contact-angle analyser, and the accuracy of the results obtained was confirmed by using a micro-balance technique. The values of the relevant physical parameters for the three liquids used are listed in Table 1. In the present work we restrict our attention to the results for two of the materials with the most extreme thermal conductivities, namely aluminium $(\mathrm{Al})\left(k^{\mathrm{s}}=237 \mathrm{~W} \mathrm{~m}^{-1} \mathrm{~K}^{-1}\right)$ and $\operatorname{PTFE}\left(k^{\mathrm{s}}=0.25 \mathrm{~W} \mathrm{~m}^{-1} \mathrm{~K}^{-1}\right)$.

Two sets of experiments were conducted.

The first set of experiments was conducted using droplets of all three liquids with volumes ranging from 0.5 to $8 \mu \mathrm{l}$ in an atmosphere of air with fixed temperature $T_{\mathrm{a}}=295 \mathrm{~K}$, pressure $p_{\mathrm{a}}=998 \mathrm{mbar}$, and relative saturation $H=0.4$ for water and $H=0$ for acetone and methanol.

The second set of experiments was conducted using only droplets of water with volume $V=2.5 \mu \mathrm{l}$ in an atmosphere of helium with fixed temperature 
$T_{\mathrm{a}}=295 \mathrm{~K}$ using an environmental chamber in which the atmospheric pressure could be varied in the range from 40 to 1000 mbar. The chamber is large enough to assume that the relative saturation far from the droplet remains zero throughout the evaporation, i.e. that $H=0$.

In all of the experiments it was found that the droplet volume $V$ decreased approximately linearly as a function of $t$ (see, for example, David et al. [11, Fig. 4]) and hence subsequently we compare values of the average evaporation rate $-\mathrm{d} V / \mathrm{d} t$ as a function of droplet radius $R$ and atmospheric pressure $p_{\mathrm{a}}$.

Figure 2 near here.

Figure 2 shows the theoretical predictions for the temperature of droplets of methanol on $\mathrm{Al}$ and PTFE substrates in an atmosphere of air. Since Al is a good conductor, the temperature of the substrate is approximately constant at the atmospheric temperature $T_{\mathrm{a}}$, and essentially all of the cooling takes place within the droplet. On the other hand, since PTFE is a poor conductor, there is significant cooling within the substrate in addition to the cooling that takes place within the droplet. This leads to significantly more cooling, and hence to a lower evaporation rate, than on a better conductor. The theoretical predictions for the evaporative cooling shown in Fig. 2 are consistent with the experimentally measured values of the bulk temperature reported by David et al. [11, Table 2], but significantly larger than those predicted by the numerical calculations of $\mathrm{Hu}$ and Larson [5, Fig. 1] for broadly similar physical situations.

Figure 3 near here.

Figure 3 shows the theoretical predictions for the concentration of vapour in an atmosphere of air for methanol droplets on $\mathrm{Al}$ and PTFE substrates. In particular, Figure 3(b) clearly shows that the saturation concentration of vapour at the free surface of the droplet is not uniform, contrary to what is assumed in the Deegan model.

Figure 4 near here.

Figure 4 shows the theoretical predictions for the local evaporative mass flux of both the present model and the Deegan model given by Eq. (13) for methanol droplets on both $\mathrm{Al}$ and PTFE substrates in an atmosphere of air. In particular, it shows that the mass flux is singular at the contact line $r=R$ in both models.

Figure 5 near here.

Figure 5 shows the comparison between the experimentally measured values 
of the average evaporation rate for droplets of all three liquids of various radii on both substrates and the corresponding theoretical predictions of both the present model and the Deegan model given by Eq. (14). Figure 5 shows that there is good qualitative agreement between the experimental and theoretical results. For acetone and methanol there is good quantitative agreement for the $\mathrm{Al}$ substrate, but the theory under-estimates the evaporation rate for the PTFE substrate. Although not readily apparent from Fig. 5, for water the theory under-estimates the evaporation rate for both substrates. Nevertheless, in view of the many assumptions made in deriving the model, the agreement is remarkably good, especially as there are no "fitting" parameters in the theory, no "fine tuning" of the values of the physical parameters has taken place, and the droplets in the experiments are not particularly thin (specifically, their contact angles are not particularly small). Perhaps the most satisfying aspect of the agreement shown in Fig. 5 is the manner in which the present model captures the significant difference in evaporation rate between droplets of the same liquid on different substrates. Figure 5 also shows that the predictions of the Deegan model are close to those of the present model for the Al but not the PTFE substrate. Again this is because Al is a much better conductor than PTFE and hence the evaporative cooling of a droplet on $\mathrm{Al}$ is much less than that of a droplet on PTFE, so that the saturation concentration of vapour at the free surface is much closer to the constant value of $c_{\text {sat }}\left(T_{\mathrm{a}}\right)$ assumed in the Deegan model.

Figure 6 near here.

Figure 6 shows the comparison between the experimentally measured values of the average evaporation rate for droplets of water on both substrates in an atmosphere of helium at various pressures and the corresponding theoretical predictions of both the present model and the generalised Deegan model given by Eq. (14) with $D$ given by Eq. (12). Figure 6 shows that there is again good qualitative agreement between the experimental and theoretical results. In particular, there is excellent quantitative agreement for the Al substrate, but poorer agreement for the PTFE substrate for which the evaporation rate is significantly under-estimated, especially at low atmospheric pressures. As before, the present model captures the significant difference in evaporation rate between droplets of the same liquid on different substrates.

\section{Discussion}

In this paper we formulated and solved a mathematical model for the quasisteady diffusion-limited evaporation of a thin axisymmetric sessile droplet of liquid with a pinned contact line which generalises the theoretical model pro- 
posed by Deegan et al. [2] to include the effect of evaporative cooling on the saturation concentration of vapour at the free surface of the droplet, and the dependence of the coefficient of diffusion of vapour in the atmosphere on the atmospheric pressure. The predictions of the model were found to be in good qualitative, and in some cases also quantitative, agreement with the recent experimental results of David et al. [11]. In particular, they capture the experimentally observed dependence of the total evaporation rate on the thermal conductivities of the liquid and the substrate, and on the atmospheric pressure.

The present initial model can, of course, be refined in several ways in order to improve the agreement between theory and experiment. Perhaps the most unrealistic assumptions in the present model are that both the droplet and the substrate are thin (and, in particular, that the contact angle $\theta$ is small). In ongoing numerical work we have relaxed these assumptions to study the general case of a non-thin droplet on a non-thin substrate, and preliminary results indicate that this generalisation improves the quantitative agreement between the theoretical predictions and experimental results for acetone and methanol in air, but still leads to an under-estimation of the evaporation rate for water in air. A possible explanation for this latter under-estimation may be, as suggested recently by Shahidzadeh-Bonn et al. [9], that because water vapour (unlike acetone or methanol vapour) is lighter than air, buoyancy effects may enhance the transport of vapour away from the droplet in this case.

We are also currently refining the model for situations (such as, for example, low atmospheric pressure) in which the evaporative cooling is sufficiently large that the simple linear relationship for the saturation concentration as a function of temperature given by Eq. (8) ceases to be sufficiently accurate. Preliminary results indicate that this improves the agreement between the theoretical predictions and the experimental results for droplets of water in a helium atmosphere on a PTFE substrate at low atmospheric pressure.

Another physical effect not included in the present model is surface-tensiongradient-driven (Marangoni) flow within the droplet (as discussed by, for example, $\mathrm{Hu}$ and Larson [5]). Comparing the relative sizes of surface-tensiondriven and surface-tension-gradient-driven flow in the governing equation for $h$ (not given here for brevity) reveals that the appropriate non-dimensional Marangoni number measuring the relative importance of the Marangoni effect is

$$
M=\frac{\lambda L D(1-H) c_{\mathrm{sat}}\left(T_{\mathrm{a}}\right)}{\theta(0) \sigma\left(T_{\mathrm{a}}\right) k}
$$

where $\sigma=\sigma(T)$ is the (in general, temperature-dependent) surface tension, 
and

$$
\lambda=-\left.\frac{\mathrm{d} \sigma}{\mathrm{d} T}\right|_{T=T_{\mathrm{a}}}
$$

is the (in general, positive) surface-tension gradient at $T=T_{\mathrm{a}}$. Using the parameter values given in Table 1 and the values $\lambda=1.12 \times 10^{-4} \mathrm{~N} \mathrm{~m}^{-1} \mathrm{~K}^{-1}$, $H=0$ and $\theta(0)=43^{\circ}$ for acetone, $\lambda=7.73 \times 10^{-5} \mathrm{~N} \mathrm{~m}^{-1} \mathrm{~K}^{-1}, H=0$ and $\theta(0)=43^{\circ}$ for methanol, and $\lambda=1.68 \times 10^{-4} \mathrm{~N} \mathrm{~m}^{-1} \mathrm{~K}^{-1}, H=0.4$ and $\theta(0)=60^{\circ}$ for water, reveals that $M=0.14$ for acetone, $M=7.6 \times 10^{-2}$ for methanol and $M=2.6 \times 10^{-3}$ for water, indicating that while Marangoni effects could play some role for droplets of acetone and methanol they are likely to negligible for droplets of water.

\section{Acknowledgements}

This work is supported by the United Kingdom Engineering and Physical Sciences Research Council via grants GR/S59444 and GR/S59451.

\section{References}

[1] R. D. Deegan, Pattern formation in drying drops, Phys. Rev. E, 61 (2000) 475-485.

[2] R. D. Deegan, O. Bakajin, T. F. Dupont, G. Huber, S. R. Nagel, T. A. Witten, Contact line deposits in an evaporating drop, Phys. Rev. E, 62 (2000) 756-765.

[3] H. Hu, R. G. Larson, Evaporation of a sessile droplet on a substrate, J. Phys. Chem. B, 106 (2002) 1334-1344.

[4] H. Hu, R. G. Larson, Analysis of the microfluid flow in an evaporating sessile droplet, Langmuir, 21 (2005) 3963-3971.

[5] H. Hu, R. G. Larson, Analysis of the effects of Marangoni stresses on the microflow in an evaporating sessile droplet, Langmuir, 21 (2005) 3972-3980.

[6] Y. O. Popov, Evaporative deposition patterns: spatial dimensions of the deposit, Phys. Rev. E, 71 (2005) 036313-1-036313-17.

[7] C. Poulard, G. Guéna, A. M. Cazabat, Diffusion-driven evaporation of sessile drops, J. Phys.: Condens. Matter, 17 (2005) S4213-S4227.

[8] E. Sultan, A. Boudaoud, M. Ben Amar, Evaporation of a thin film: diffusion of the vapour and Marangoni instabilities, J. Fluid Mech., 543 (2005) 183-202. 
[9] N. Shahidzadeh-Bonn, S. Rafaï, A. Azouni, D. Bonn, Evaporating droplets, J. Fluid Mech., 549 (2006) 307-313.

[10] F. Girard, M. Antoni, S. Faure, A. Steinchen, Evaporation and Marangoni driven convection in small heated water droplets, Langmuir, 22 (2006) 1108511091.

[11] S. David, K. Sefiane, L. Tadrist, "Experimental investigation of the effect of thermal properties of the substrate in the wetting and evaporation of sessile drops," Colloids and Surfaces A: Physicochem. Eng. Aspects, 298 (2007) 108114 .

[12] G. J. Dunn, S. K. Wilson, B. R. Duffy, S. David, K. Sefiane, The effect of the thermal conductivity of the substrate on droplet evaporation, to appear in the proceedings of the 14th European Conference on Mathematics for Industry (ECMI 2006), 10th-14th July 2006, Madrid, Spain, Springer, 2007.

[13] R. C. Reid, J. M. Prausnitz, B. E. Poling, The Properties of Liquids and Gases, 4th ed., McGraw-Hill, 1987. 


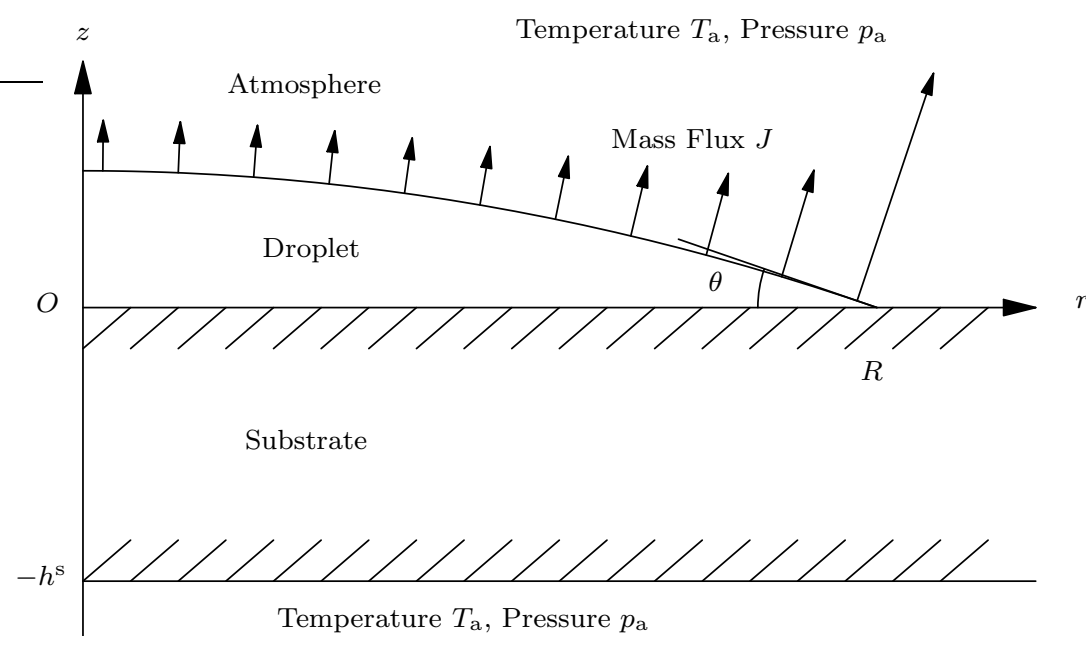

Fig. 1. Geometry of the problem. 

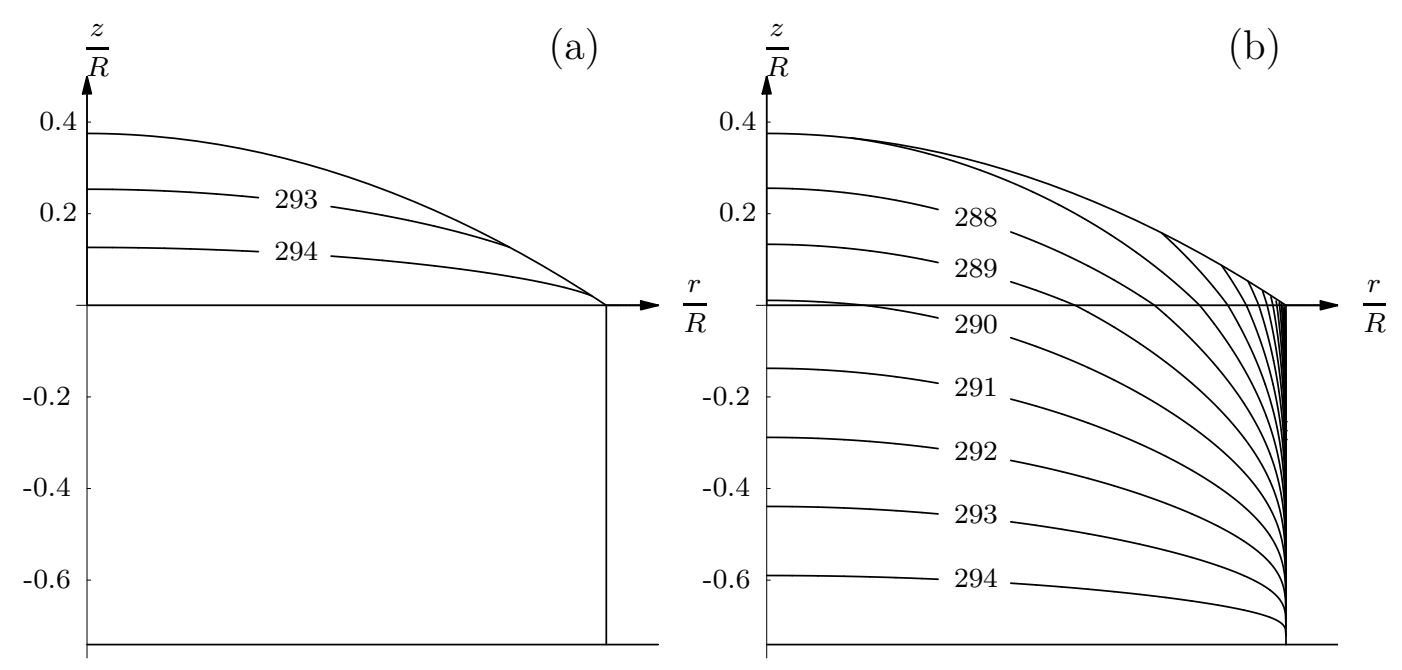

Fig. 2. Theoretical predictions for the temperature of droplets of methanol with radius $R=1.35 \mathrm{~mm}$ and contact angle $\theta=43^{\circ}$ on (a) Al and (b) PTFE substrates in an atmosphere of air at temperature $T_{\mathrm{a}}=295 \mathrm{~K}$ and pressure $p_{\mathrm{a}}=998 \mathrm{mbar}$. 

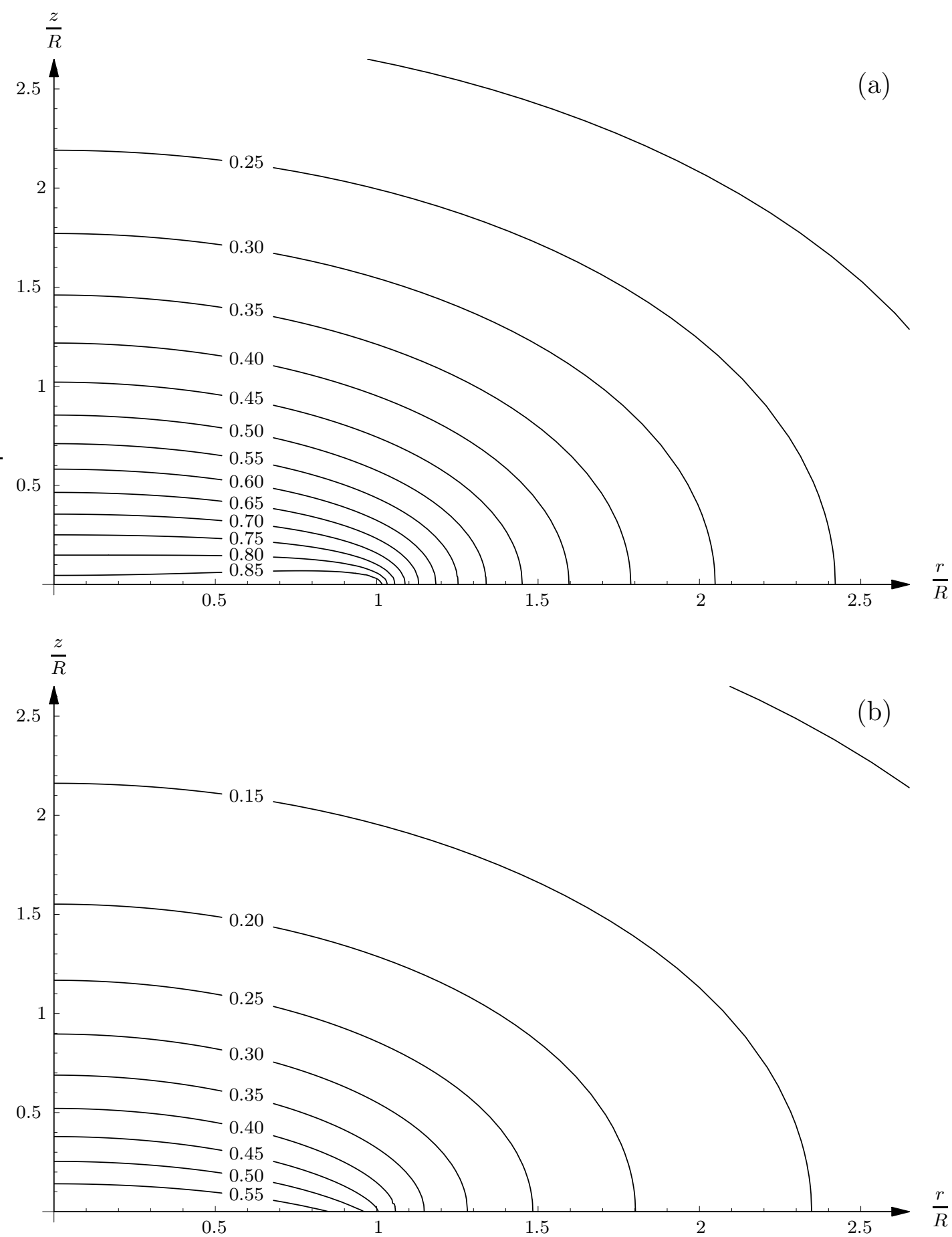

Fig. 3. Theoretical predictions for the concentration of vapour in an atmosphere of air at temperature $T_{\mathrm{a}}=295 \mathrm{~K}$ and pressure $p_{\mathrm{a}}=998 \mathrm{mbar}$ for methanol droplets of radius $R=1.35 \mathrm{~mm}$ and contact angle $\theta=43^{\circ}$ on (a) Al and (b) PTFE substrates. The contours are labelled with the appropriate (non-dimensional) values of $c / c_{\mathrm{sat}}\left(T_{\mathrm{a}}\right)$. 


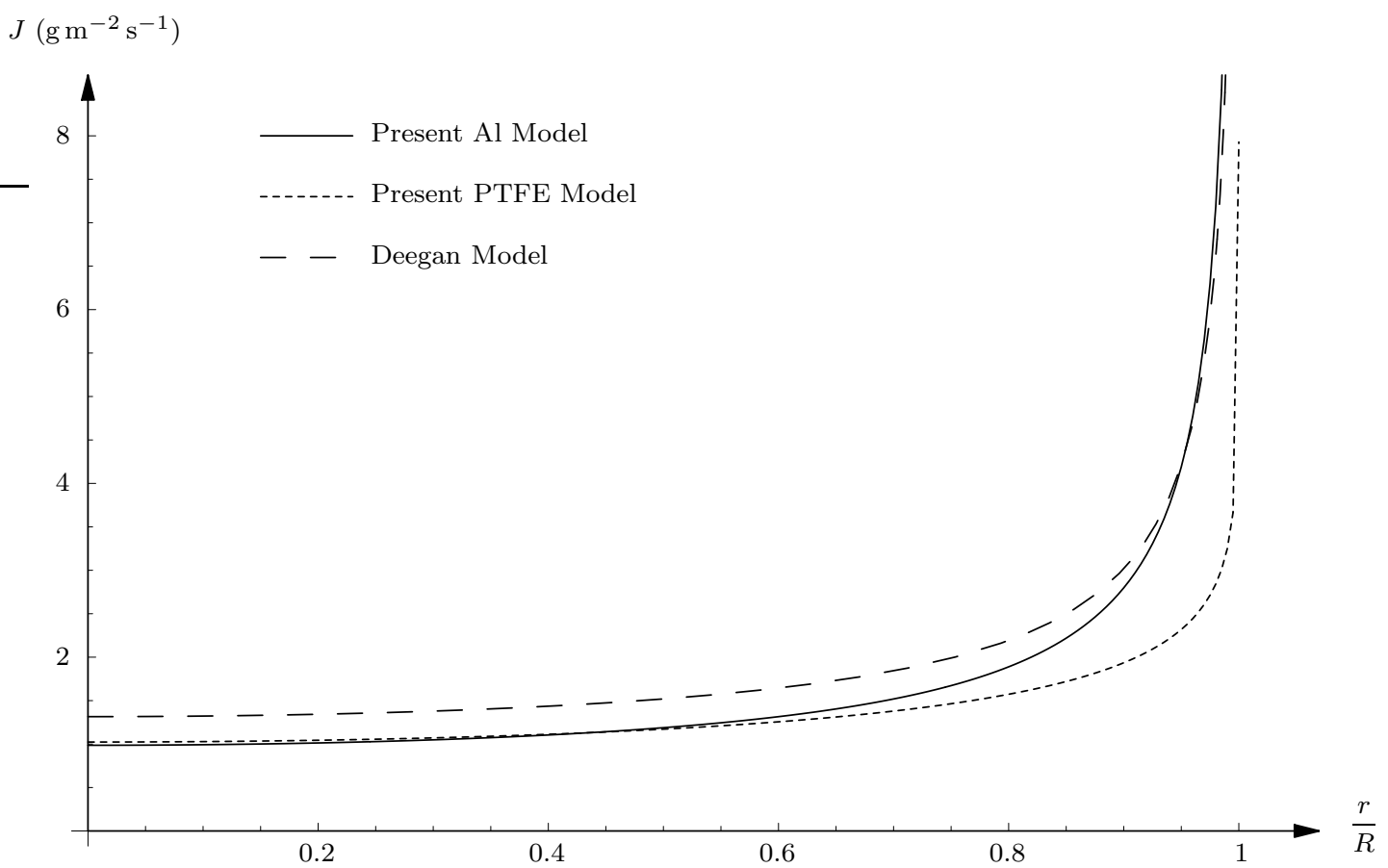

Fig. 4. Theoretical predictions for the local evaporative mass flux of both the present model and the Deegan model given by Eq. (13) for methanol droplets of radius $R=1.35 \mathrm{~mm}$ and contact angle $\theta=43^{\circ}$ on both $\mathrm{Al}$ and PTFE substrates in an atmosphere of air at temperature $T_{\mathrm{a}}=295 \mathrm{~K}$ and pressure $p_{\mathrm{a}}=998 \mathrm{mbar}$. 


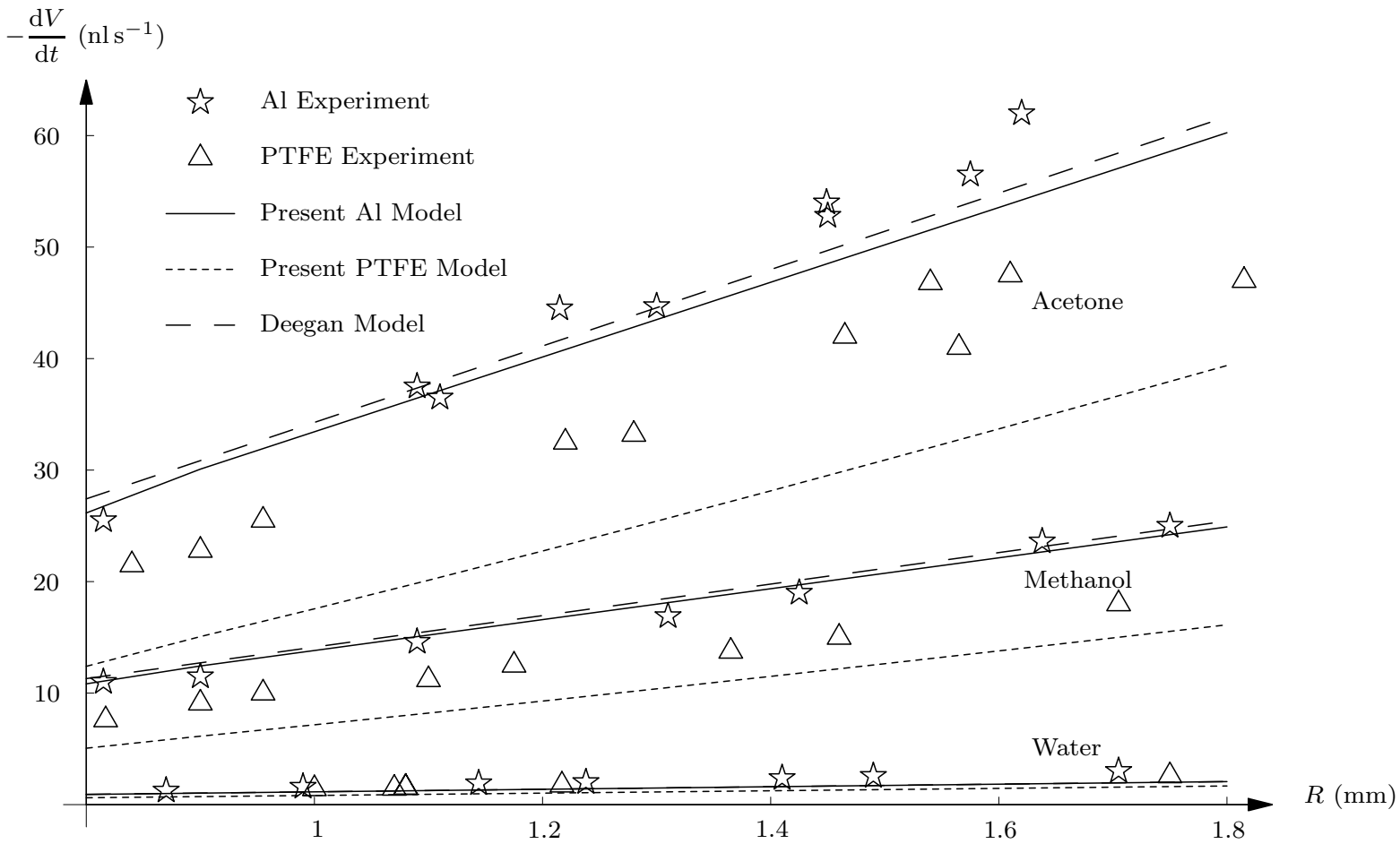

Fig. 5. Comparison between the experimentally measured values of the average evaporation rate for droplets of all three liquids of various radii on both substrates and the corresponding theoretical predictions of both the present model and the Deegan model given by Eq. (14) in an atmosphere of air. 


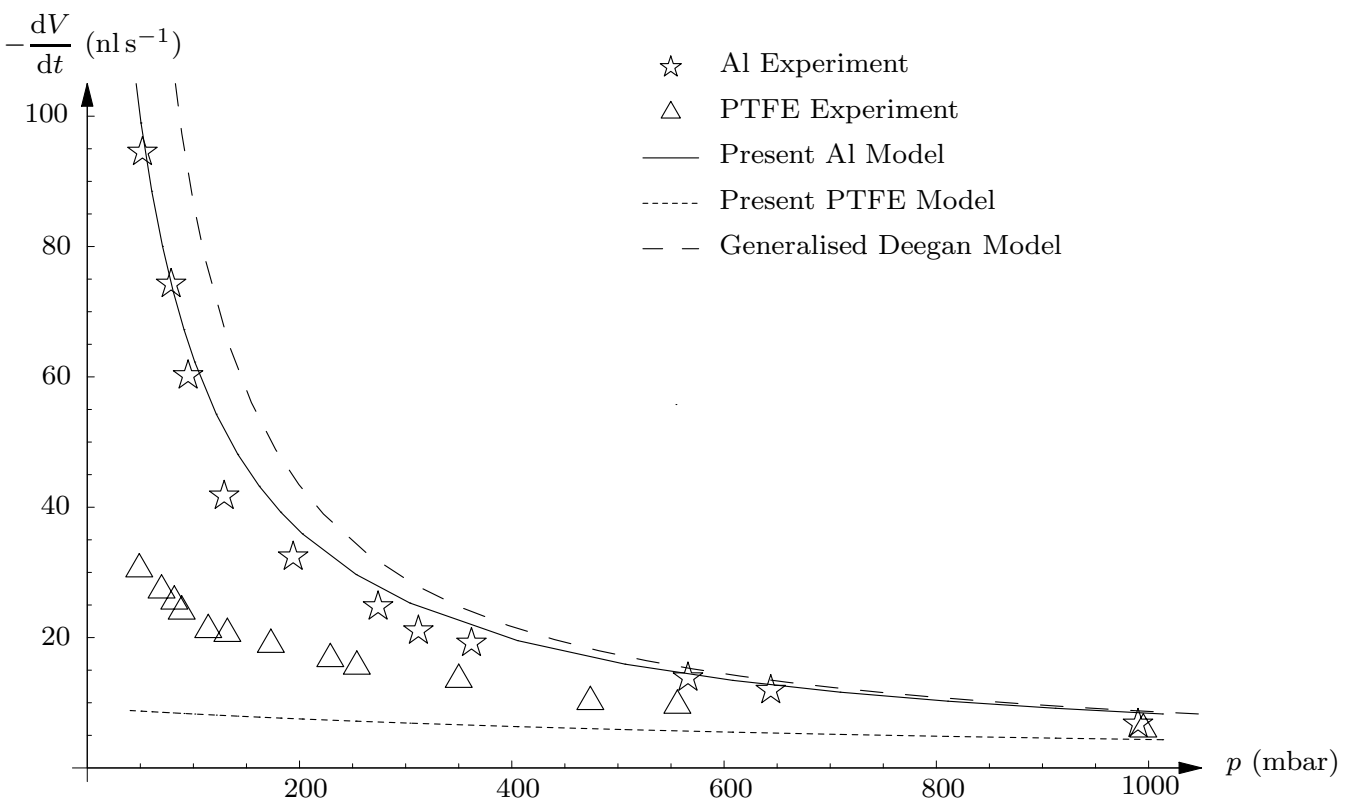

Fig. 6. Comparison between the experimentally measured values of the average evaporation rate for droplets of water on both substrates in an atmosphere of helium at various atmospheric pressures and the corresponding theoretical predictions of both the present model and the generalised Deegan model given by Eq. (14) with $D$ given by Eq. (12). 